\title{
Wie viele Tempora hat das Deutsche?
}

\author{
Abraham P. ten Cate \\ Afdeling Duitse taal en cultuur, Rijksuniversiteit Groningen, Nederland
}

This contribution deals with the question of how many tenses exist in the German language. Answers to this question range from a minimum of one up to no less than about thirteen, but the main problem is, what exactly has to be counted. One has to distinguish between tense forms and temporal functions, and standard German has about 6 forms and 6 functions. Almost every form expresses more than one function, so that about 12 pairs of form and function may be found. Such overlap is not necessary, because speakers can avoid using two forms for one function. However, since this situation is not excluded in principle, we have to accept a certain redundancy in the German tense system.

\section{VORBEMERKUNGEN}

Wer sich mit der Tempuskategorie befasst, hat immer vorab klar zu stellen, welcher Tempusbegriff zugrundegelegt wird. Einige Annahmen, von denen diese Arbeit mehr oder weniger axiomatisch ausgeht (s. für Begründungen u.a. ten Cate 2004), sind erstens, dass in dem deutschen Tempussystem der Aspekt keine Rolle spielt, zweitens, dass Tempusfunktionen auf den Indikativ beschränkt sind, da nur die Indikativformen deiktisch, d.h. in der Sprechzeit verankert sind und, drittens, dass die temporalen Relationen durch ein Koordinatensystem zu definieren sind, dem drei so genannte Orientierungspunkte zu Grunde gelegt sind, nämlich (a) der Zeitpunkt, zu dem, oder das Intervall, in dem die Sachverhaltsbeschreibung geäußert wurde, also die Sprechzeit tS; (b) der Zeitpunkt, zu dem, oder das Intervall, in dem der beschriebene Sachverhalt stattfindet, also die Aktzeit tA, bzw. die Eventzeit tE; und (c) der Zeitpunkt oder das Intervall, von dem aus der beschriebene Sachverhalt betrachtet wird, also die Referenzzeit tR. Die Frage, ob tR für die Beschreibung der temporalen Struktur aller Tempora gebraucht wird, oder ob die Referenzzeit nur für bestimmte Tempora, wie etwa das Plusquamperfektum und das Futurum exactum, notwendig ist, soll in diesem Zusammenhang nicht erörtert werden.

Die Frage scheint berechtigt, wozu das Zählen von Tempora gut sein soll. Die Antwort könnte ungefähr so lauten: Wenn man sich bemüht die Zahl der Tempora in einer Sprache festzulegen, stößt man auf Fragen, die mit den begrifflichen Grenzbereichen zu tun haben. Was soll als Tempus gezählt werden und was nicht? Auch der Begriff 'Deutsch' ist leider nicht so homogen, wie man ihn haben möchte: Gibt es einen eindeutigen Standard im Bereich der Tempora? Sind z.B. das doppelte Perfekt und Plusquamperfekt (etwa in 1a und b) auch in einer Beschreibung der Standardsprache zu 
berücksichtigen?

1a) Ich habe meinen Bruder lange nicht besucht gehabt.

1b) Ich hatte meinen Bruder lange nicht besucht gehabt.

Und wie verhalten sich Perfekt und Präteritum zueinander? Sind sie voneinander zu unterscheiden, wenn sie einander ohne weiteres ersetzen oder wenn der Gebrauch der einen Form zur Aufgabe der anderen führt? Ziemlich kompliziert ist auch die Relation zwischen Tempusform und -funktion, die, obwohl von hervorragender Bedeutung, im Allgemeinen unberücksichtigt geblieben ist. Es wird sich zeigen, dass eine exakte Antwort auf die Titelfrage von geringerem Belang ist als die Diskussion, zu der sie Anlass gibt.

\section{Von 13 TeMPORA ZU 1}

Die nun folgende Behandlung der gängigsten Tempussysteme folgt Thieroff (1992: 4664), der eine gediegene Übersicht gibt, basiert aber zum Teil auch auf neueren Arbeiten. $\mathrm{Zu}$ unterscheiden sind Tempusmodelle mit unterschiedlichen Tempuszahlen.

A.

Wenn neun oder mehr Tempora angenommen werden, so entspringt dies nach Thieroff (1992: 51) Bemühungen, zunächst ein 'ideales' System zu skizzieren und danach zu überprüfen, welche Stellen in diesem System in einer Einzelsprache besetzt sind. Allgemein gilt für diese Systeme, dass die drei Zeitstufen Gegenwart, Vergangenheit und Zukunft jeweils drei Tempora kennen, nämlich Gleich-, Vor- und Nachzeitigkeit, siehe (2):

2) Neun Tempora

\begin{tabular}{l|l|l|l} 
& Gegenwart & Vergangenheit & Zukunft \\
\hline Gleichzeitigkeit & Präsens & Präteritum & Futur I (werden + Inf.) \\
\hline Vorzeitigkeit & Perfekt & Plusquamperfekt & $\begin{array}{l}\text { Futur II (wird + 2. Part. + } \\
\text { haben/sein) }\end{array}$ \\
\hline Nachzeitigkeit & wollen/werden-Futur & wollte/sollte-Futur & $\begin{array}{l}\text { werden und } \\
\text { wollen/sollen-Futur }\end{array}$
\end{tabular}

Gegenwartstempora sind dann Präsens, Perfekt und will/werden-Futur, Vergangenheitstempora das Präteritum, Plusquamperfekt und das Vergangenheitsfutur mit wollte/sollte, und Zukunftstempora das werden-Futur, Futur II und ein werden + wollen/sollen-Futur. Ein mysteriöser Punkt ist das zweimalige Vorkommen des werdenFuturs, nämlich einmal als futurische Gegenwart und zum zweiten als präsentische Zukunft. Es fragt sich sehr, ob diese Dichotomie einer Zweideutigkeit des werden-Futurs entspricht, oder ob sie aus Symmetriegründen, also aus einem gewissen grammatischen Systemzwang erklärt werden soll: Eine Eliminierung dieser Ungereimtheit führt an sich schon zu einem 8-Temporasystem. 
Auch Reichenbachs (1947: 297) Tempussystem gehört in die Kategorie der temporalen Systeme, denen eine 'ideale' temporale Matrix zu Grunde liegt. Die drei Orientierungspunkte tS, tA und tR ergeben insgesamt die in (3) aufgeführten 13 verschiedenen Konstellationen. Thieroff (1992: 54) übernimmt zwar Reichenbachs Orientierungspunkte, ordnet aber im Übrigen Reichenbachs Tempusklassifizierung den "Konstrukten von Philosophen" zu, die er von der Hand weist, wobei sich die Kritik im Wesentlichen auf das Vorkommen von zwei zu Grunde liegenden Funktionen für das Futur richtet. Reichenbachs Namengebung unter New Name geht nach einem bewundernswert einfachen Prinzip vor, in Reichenbachs (1947: 297, Übersetzung APtC) Worten: "Die Positionierung von $R$ relativ zu $S$ wird mit den Wörtern 'past', 'present' und 'future' angegeben. Die Positionierung von $E$ relativ zu R wird wiedergegeben mit den Wörtern 'anterior', 'simple' und 'posterior', wobei 'simple' das Zusammenfallen von $R$ und $E$ bezeichnet." Das führt zu dem Schema in (3), in dem die traditionellen Bezeichnungen hier um die deutschen Begriffe ergänzt wurden:

3) Tempora nach Reichenbach (1947)

\begin{tabular}{|c|c|c|c|c|}
\hline$\#$ & Structure & New Name & Traditional Name & $\begin{array}{l}\text { Traditionelle } \\
\text { Bezeichnung }\end{array}$ \\
\hline 1 & E-R-S & Anterior past & Past perfect & Plusquamperfekt \\
\hline 2 & $E, R-S$ & Simple past & Simple past & Präteritum \\
\hline 3 & $\mathrm{R}-\mathrm{E}-\mathrm{S})$ & & & \\
\hline 4 & $\mathrm{R}-\mathrm{S}, \mathrm{E}$ & Posterior past & - & - \\
\hline 5 & $\mathrm{R}-\mathrm{S}-\mathrm{E}$ & & & \\
\hline 6 & E-S, R & Anterior present & Present perfect & Präsensperfekt \\
\hline 7 & $S, R, E$ & Simple present & Present & Präsens \\
\hline 8 & S, R-E & Posterior present & Simple future & Futur I \\
\hline 9 & $S-E-R$ & & & \\
\hline 10 & $\mathrm{~S}, \mathrm{E}-\mathrm{R}$ & Anterior future & Future perfect & Futur II \\
\hline 11 & $\mathrm{E}-\mathrm{S}-\mathrm{R}$ & & & \\
\hline 12 & $S-R, E$ & Simple future & Simple future & Futur I (?) \\
\hline 13 & S-R-E & Posterior future & - & - \\
\hline
\end{tabular}

B.

Wie unter A. gezeigt wurde, kann ein System mit neun Tempora einfach zu einem System mit acht Tempora reduziert werden. Auch Janssen (1994: 94) erörtert ein 8-TemporaSystem, das er als maßgeblich für Beschreibungen des Niederländischen betrachtet. Es hat neben den üblichen Tempora nicht weniger als 4 Futurtempora, die hier unter (4) aufgeführt sind:

4) Futurum nach Janssen (1994):

futurum (zal vertrekken; wird abreisen);

futurum exactum (zal zijn vertrokken; wird abgereist sein); 
futurum praeteriti (zou vertrekken; würde/wollte/sollte abreisen); und futurum exactum praeteriti (zou zijn vertrokken; würde/sollte abgereist sein).

In Beschreibungen des Deutschen werden die beiden letzten Formen nicht unbedingt immer als Tempora gesehen, da sie, anders als ihre niederländischen Entsprechungen, Modalverben enthalten, $\mathrm{nl}$. wollte/sollte abreisen und sollte abgereist sein. Im Übrigen kommt Janssen selbst schließlich zu einem 2-Tempora-System, worauf noch zurückzukommen ist.

C.

Ein System mit sieben Tempora wird u.a. von Zifonun u.a. (1997: 1712f.) vorgelegt. Es umfasst 3 einfache (Präsens, Präteritum, werden-Futur) und 4 zusammengesetzte Tempora, nämlich Infinitiv Perfekt (z.B. bestiegen haben in (5)), Präsensperfekt, Präteritumperfekt und Futurperfekt.

5) Peter will den Schneekofel bestiegen haben.

D.

Modelle mit sechs Tempora sind in Grammatiken gut vertreten, z.B. in der DudenGrammatik (1998). Ten Cate et al. (1998) hat eigene Paragrafen für Präsens, Perfekt, Präteritum, Plusquamperfekt und Futur (I + II), also ebenfalls sechs, aber es wird ausgeführt, dass die Formen mehrere Funktionen haben können, nämlich 4 beim Präsens, 3 beim Perfekt, eine bei Präteritum und Plusquamperfekt (gelegentlich aber 2, nämlich beim Plusquamperfektum pro Praeterito) und 3 Futurtempora (nämlich Futur I + II und sollen/wollen), maximal also nicht weniger als dreizehn sogenannte FormFunktionspaare.

E.

In einem eigenen Paragrafen unter dem Titel "5-4-3-2-1 Tempora oder Das Deutsche auf dem Weg zur tempuslosen Sprache" schildert Thieroff (1992: 62), wie "in jüngerer und jüngster Zeit in einer zunehmenden Zahl von Arbeiten zu den deutschen Tempora der Status einer Tempus-Kategorie für immer mehr Formen bestritten" wird. Dies betrifft dann z.B. das Futur II, das als ein Latinismus gesehen wird, der nicht zum guten Deutsch gehört, oder das Futur überhaupt, weil es eher in die Modalität als in das Tempus gehört. Dies ist beispielsweise in der surinamischen Variante des Niederländischen auch faktisch der Fall, zumindest was das analytische Futur betrifft, da eine durch zullen (werden) + Infinitiv ausgedrückte Zukunft als ungewisser empfunden wird als ein durch die Präsensform ausgedrücktes Futur (de Kleine 2002). Es resultieren dadurch 5, bzw. 4 Tempora. Wenn die so genannten 'Perfekttempora' als Aspektvarianten gesehen werden, reduziert sich die Zahl auf drei. Eine interessante 2-Tempora-Hypothese ist die von Theo Janssen (1994), der die Tempora auf Präsens und Präteritum reduziert, da diese die einzigen Tempusvarianten sind, die durch analytische Verbformen zum Ausdruck 
gebracht werden. Die Begründung dafür ist im Wesentlichen, dass nur finite Verbformen auf die Sprechzeit Bezug nehmen (Janssen 1994: 103). Dadurch werden praktisch die analytischen Tempora eliminiert: Janssen ordnet dem 2. Partizip eine temporale Lesart zu, die dann mit einem finiten Verb im Präsens oder Präteritum verträglich ist.

Thieroff nennt noch den Vorschlag von Mugler (1988), demzufolge nur das Präteritum ein Tempus ist, da das Präsens als merkmallose default-Form eingestuft wird.

\section{Form-FunkTIONSPAARE}

Die wissenschaftlich interessanten Vorschläge sind zunächst die von Reichenbach (1947) und Janssen (1994). Sie basieren beide auf einer ausgeklügelten Systematik, sind auch beide hochgradig radikal, kommen aber zu sehr unterschiedlichen Ergebnissen. Reichenbachs Modell ist sehr attraktiv: Ausgehend von den drei Orientierungspunkten wird untersucht, welche Konstellationen theoretisch, also rein rechnerisch, möglich sind, und in einem zweiten Durchgang wird überprüft, welche Funktionen in einer Sprache eine formale Entsprechung haben. Wenden wir uns einmal den Kombinationen R-E-S; R-S, E; und R-S-E zu, die in Reichenbachs Terminologie die Funktion Posterior Past zum Ausdruck bringen, die aber keine traditionelle Bezeichnung haben: Bei RE-S wird ein Vorgang beschrieben, der vor dem Sprechzeitpunkt liegt, aber nach der Betrachtzeit. Diese Kombination liegt vor in einer Beschreibung wie (6):

6) Er sollte seine Heimat nie wiedersehen.

Für diese Art von temporalen Verhältnisse gibt es keinen anerkannten traditionellen Terminus, man hilft manchmal mit Begriffen wie 'Schicksalsfügung' oder 'Zukunft in der Vergangenheit' aus. Reichenbachs Modell kann genau beschreiben, welche Konstellation von Orientierungspunkten diese Lesart bedingt. Satz (6) ist aber trotzdem kein gutes Beispiel, da er eigentlich komplex ist, wenn er auch nur eine finite Verbform enthält, denn sollen ist nicht ein temporales Auxiliar. R-S, E liegt vor, wenn in der 'Schicksalslesart ' das Ereignis zeitlich mit der Sprechzeit zusammenfällt. Eine mögliche, allerdings aber ebenfalls eindeutig komplexe Sachverhaltsbeschreibung zu dieser Konstellation dürfte (7) sein:

7) Meine Mutter hatte nie gedacht, dass ich noch einmal Linguist werden sollte.

Die dritte Konstellation, R-S-E, ist noch problematischer. Hier soll ein Sachverhalt beschrieben werden, der von der Sprechzeit aus gesehen in der Zukunft liegt, der aber von einem Orientierungspunkt $\mathrm{R}$ aus betrachtet wird, der von tS aus betrachtet in der Vergangenheit liegt. Eine mögliche Sachverhaltsbeschreibung, die dazu passt, liegt vor in (8):

8) Schon Kopernikus wusste, dass die Welt einmal untergeht.

Sowohl in (7) wie in (8) werden zwei finite Verbformen gebraucht um ein temporales Verhältnis zu beschreiben, so dass diese Beispiele außer ihrem didaktischen Sinn 
ebenfalls innerhalb der Tempussystematik nicht adäquat sind.

In traditionellen Tempusübersichten gibt es auch nicht so etwas wie Posterior Future, die Konstellation S-R-E. In diesem Fall wird von einem Betrachtzeitpunkt tR aus ein Vorfall beschrieben, der sowohl nach tS wie nach tR stattfindet, wobei aber tR zeitlich nach tS angesetzt ist, eine Situation, der (9) in etwas entspricht:

9) Morgen wird der Urlaubsbeginn nur noch einen Tag von uns entfernt sein.

Etwas wunderlich sind die beiden Konstellationen 8 und 12 in Tafel (3), die eine genaue Parallele zu Tafel (1) aufweisen: Die Konstellation 12 ist eine rein theoretische, denn es widerspricht dem Präsenscharakter der Verbform, dass der Betrachtzeitpunkt von der Sprechzeit losgelöst und in die Zukunft verlagert wird, obwohl so etwas auch bei dem angeblich 'unnatürlichen' Futur II festzustellen ist.

Reichenbachs Modell gibt Zusammengehörigkeitsbeziehungen von Tempora besonders schön wieder: Plusquamperfekt und Präteritum zeichnen sich dadurch aus, dass bei beiden sowohl E wie R vor S angesetzt sind; das Gemeinsame an Präsensperfekt, Präsens und Futur I ist ihre Gegenwartsbezogenheit, denn S und R fallen bei ihnen zusammen. Futur I und Präsensperfekt sind in gewissem Sinne spiegelbildliche Tempora, da beim Futur I tA den beiden Orientierungspunkten tS und tR folgt, aber bei Präsensperfekt ihnen vorangeht.

\section{Erweiterung der Form-/Funktionspaare?}

Welche Beziehungen zwischen Formen und Funktionen können festgestellt werden?

Reichenbach nimmt auffälligerweise eine 1:1-Entsprechung von Form und Funktion an, d.h. für den Ausdruck jeder Funktion wird genau eine Form gefunden. Das ist aber eine zu einfache Wiedergabe der wirklichen Verhältnisse, denn die meisten Formen erfüllen mehr als eine Funktion, mit dem Präsens als unbezweifelbarem Meister, da das Präsens die meisten Funktionen zum Ausdruck bringt: nach ten Cate et al. (1998: 85) das aktuelle Präsens (10), das generelle Präsens (11), das futurische Präsens (12) und das historische Präsens (13):

10) Schwitzend liest der Redner seinen Vortrag vor.

11) Brabrand liegt in der Umgebung von Århus.

12) Wir kommen wieder!

13) Die Reise der Nibelungen endet in Ungarn.

Zwischen dem aktuellen und dem generellen Präsens gibt es keinen deiktischen Unterschied, die beiden Präsensarten unterscheiden sich nur durch die Dauer des beschriebenen Sachverhalts, wodurch die Zahl der präsentischen Form-Funktionspaare auf drei kommt. Das Perfekt hat ebenfalls drei solcher Paare (Präsensperfekt, Futurperfekt und Perfektum pro Praeterito); das Präteritum praktisch nur eines, wenn man relativ seltene Präsensvarianten wie (14) und (15) außer Betracht lässt: 
14) Du warst der Prinz.

15) Wie war doch Ihr Name, bitte?

Auch Futur I und II sind temporal monofunktional; das Plusquamperfekt hat zwei temporale Funktionen, nämlich Plusquamperfektund Plusquamperfektum pro Praeterito: Insgesamt kommt man also, wenn von dem doppelten Perfekt und dem doppelten Plusquamperfekt abgesehen wird, vorläufig auf 11 temporale Form-Funktionspaare. Wenn man diese Zahl mit derjenigen der sechs möglichen Funktionen und den ebenfalls sechs Formen (Präsens, Perfekt, Präteritum, Plusquamperfekt, Futur I und II) vergleicht, stellt man gleich das Maß der Überlappung fest. Dabei ergibt sich eine Reihe von ungewissen Faktoren: Schließen sich etwa die Funktionen des Präsensperfekt und des Perfektums pro Praeterito sowie das Plusquamperfekt und das Plusquamperfektum pro Praeterito gegenseitig aus, dann reduziert sich die Zahl der Paare um zwei auf neun. In den 'pro-Praeterito'-fällen ist offensichtlich auch das Präteritum zu streichen, wodurch dann noch acht übrig bleiben, aber das trifft wahrscheinlich nicht für alle Sprecher und Sprecherinnen des Deutschen und möglicherweise auch nicht für alle Verbkategorien zu. Die beiden Funktionen, die über die Formenzahl hinausgehen, sind das futurische und das historische Präsens. Wenn auch das historische Präsens funktional mit dem Präteritum zusammenfällt, so heißt das nicht, dass sie sich abwechselnd ersetzen könnten. Diese Diskussion fasst Tafel (16) zusammen:

16) Temporale Form-/Funktionspaare im Deutschen

\begin{tabular}{|c|c|c|c|c|c|c|}
\hline $\begin{array}{l}\text { Funktion } \rightarrow \\
\text { Form } \downarrow\end{array}$ & $\begin{array}{l}\text { Präsens } \\
\text { tSRA }\end{array}$ & $\begin{array}{l}\text { Präs- } \\
\text { Perf } \\
\text { tA-tSR }\end{array}$ & $\begin{array}{l}\text { Prät } \\
\text { tAR-tS }\end{array}$ & $\begin{array}{l}\text { Prät- } \\
\text { Perf } \\
\text { tA-tR-tS }\end{array}$ & $\begin{array}{l}\text { Futur I } \\
\text { tSR-tA }\end{array}$ & $\begin{array}{l}\text { Futur II } \\
\text { tS-tA-tR }\end{array}$ \\
\hline Präsens & + & & + & & + & \\
\hline Perfekt & & + & + & & & + \\
\hline Präteritum & + & (?) & + & & & \\
\hline $\begin{array}{l}\text { Plusquam- } \\
\text { perfektum }\end{array}$ & & & + & + & & \\
\hline Fut.werden & & & & & + & \\
\hline $\begin{array}{l}\text { FuturPerf. } \\
\text { (exactum) }\end{array}$ & & & & & & + \\
\hline $\begin{array}{l}\text { Doppeltes } \\
\text { Perfekt }\end{array}$ & & (?) & & & & \\
\hline $\begin{array}{l}\text { Doppeltes } \\
\text { Plusquamp. }\end{array}$ & & & & (?) & & \\
\hline
\end{tabular}

In diese Tafel sind die doppelten Perfekt- und Plusquamperfektformen nur aus Vollständigkeitsgründen aufgenommen: Ihr Status in der Standardsprache ist nämlich zweifelhaft und, wenn sie vorkommen, ist ihre temporale Funktion unsicher. Sie scheinen als Varianten der entsprechenden 'einfachen' Formen aufzutreten, übernehmen aber anscheinend auch die Funktionen dieser Formen in Varianten des Deutschen, in denen Perfekt und/oder Plusquamperfekt mit "pro-Praeterito"-Funktion auftreten. 


\section{SChlussfolgerungen}

Resümierend lässt sich feststellen, dass die Zahl der Tempora nicht eindeutig festliegt, und das schon gar nicht, wenn man sich nicht auf Tempusformen oder Tempusfunktionen beschränkt, sondern von Form-Funktionspaaren ausgeht: Sie liegt dann, sprecherabhängig, zwischen 13 und 8. Dabei sind vom funktionalen Gesichtspunkt aus Doppel-, Drei- und sogar Vierfachbesetzungen festzustellen, aber bei einem einzelnen Sprecher oder einer einzelnen Sprecherin werden solche Anhäufungen nicht vorkommen: Die Funktion Präteritum kommt bei jeder Sprecherin oder jedem Sprecher wohl mit mindesten 2 Formrepräsentationen vor, aber bei kaum einer oder einem dürfte die Zahl drei überschritten werden.

Sowohl rein funktional wie auch formal liegt die Zahl bei 6, wenn man in beiden Fällen die doppelten Perfektformen nicht mitzählt.

\section{LITERATUR}

ten Cate, Abraham P. (2004), "Descriptions of past events in German", in: Hollebrandse, Bart, Angeliek M.H. van Hout and Co Vet (eds.) (erscheint 2004), Crosslinguistic Views on Aspect, Tense and Mood, Amsterdam, New York: Rodopi.

ten Cate, Abraham P., Hans G. Lodder, André Kootte (1998), Deutsche Grammatik. Eine kontrastiv deutsch-niederländische Beschreibung für den Fremdspracherwerb, Bussum: Coutinho (2. Auflage 2004).

Duden-Grammatik (1998): Eisenberg, Peter et al., Mannheim: Duden.

Janssen, Theo A.J.M. (1994), "Tense in Dutch: Eight 'Tenses' or Two Tenses?", in: Thieroff, Rolf, Joachim Ballweg (eds.) (1994), Tense Systems in European Languages, Tübingen: Niemeyer, pp. 93-118.

de Kleine, Christa (2002), "Surinamese Dutch", in: Carlin, Eithne B., Jacques Arends (eds.) (2002), Atlas of the languages of Surinam, Leiden: KITLV Press, pp. 209-230.

Mugler, Alfred (1988), Tempus und Aspekt als Zeitbeziehungen, München: Fink.

Reichenbach, Hans (1947), Elements of symbolic logic, New York: Macmillan.

Thieroff, Rolf (1992), Das finite Verb im Deutschen. Tempus-Modus-Distanz, Tübingen: Narr.

Zifonun, Gisela u.a. (1997), Grammatik der deutschen Sprache, Berlin: de Gruyter. 